Proceedings of the 2007 Winter Simulation Conference

S. G. Henderson, B. Biller, M.-H. Hsieh, J. Shortle, J. D. Tew, and R. R. Barton, eds.

\title{
ESTIMATING TRANCHE SPREADS BY LOSS PROCESS SIMULATION
}

\author{
Kay Giesecke \\ Baeho Kim \\ Department of Management Science and Engineering \\ 414 Terman Center, Stanford University \\ Stanford, CA 94305, U.S.A.
}

\begin{abstract}
A credit derivative is a path dependent contingent claim on the aggregate loss in a portfolio of credit sensitive securities. We estimate the value of a credit derivative by Monte Carlo simulation of the affine point process that models the loss. We consider two algorithms that exploit the direct specification of the loss process in terms of an intensity. One algorithm is based on the simulation of intensity paths. Here discretization introduces bias into the results. The other algorithm facilitates exact simulation of default times and generates an unbiased estimator of the derivative price. We implement the algorithms to value index and tranche swaps, and we calibrate the loss process to quotes on the CDX North America High Yield index.
\end{abstract}

\section{INTRODUCTION}

A credit derivative is a path dependent contingent claim on the aggregate loss due to default in a portfolio of credit sensitive securities such as loans, bonds or credit swaps. The loss follows a stochastic point process that jumps at a default by the magnitude of the loss. In this paper, we show how to estimate the value of a credit derivative by Monte Carlo simulation of the loss process. To illustrate this approach, we implement the simulation based valuation and market calibration for index and tranche swaps.

We adopt the top down approach described in Giesecke and Goldberg (2005) and specify the loss process directly in terms of an intensity that represents the conditional default rate and a distribution of the loss magnitudes. We assume that the intensity follows a mean-reverting square-root jumpdiffusion process whose jump term is the loss process itself. Therefore past defaults and their recoveries influence future loss dynamics: the loss process is self-affecting and incorporates the negative dependence between default and recovery rates that is observed in credit markets.

We discuss two simulation algorithms that exploit the intensity based specification of the loss process. One algo- rithm is based on the time change theorem of Meyer (1971), which implies that under mild conditions, a general point process can be time-changed into a standard Poisson process. The time change is given by the cumulative intensity (the compensator). We use the converse of this result and simulate default times by re-scaling Poisson arrivals. This approach requires the generation of intensity paths, which is time-consuming and introduces discretization bias. This bias can be avoided with an exact algorithm that does not require path simulation. The exact algorithm is based on the observation that given a default time $T^{k}$, the waiting time to the next default is the first arrival time of a counting process started at $T^{k}$. The conditional distribution of the waiting time can be explicitly calculated as a function of the intensity at $T^{k}$, and the waiting time can be sampled directly from this distribution.

The algorithms are valid for the entire class of affine point processes specified in Errais, Giesecke, and Goldberg (2006). They can be adapted to simulate many other intensity based top down models, including the specifications in Arnsdorf and Halperin (2007), Brigo, Pallavicini, and Torresetti (2006), Davis and Lo (2001), Ding, Giesecke, and Tomecek (2006), Giesecke and Tomecek (2005), Longstaff and Rajan (2006), Lopatin and Misirpashaev (2007) and Tavella and Krekel (2006). In some situations simulation is preferred to the (semi-) analytical, characteristic function or PDE methods that are used in the top down modeling literature. For example, simulation facilitates the study of instruments with complex path-dependent payoff profiles such as cash collateralized debt obligations, which are difficult to analyze with other methods.

Duffie and Singleton (1998) and Duffie and Garleanu (2001) use similar algorithms to simulate bottom up doubly stochastic models of the individual portfolio constituents. Using a direct specification of the portfolio loss, we avoid the separate simulation of the different constituent models. This leads to a significant reduction of the computational effort in case we are interested only in derivatives on the aggregate loss but not the constituents. 


\section{Giesecke and Kim}

This article is structured as follows. In Section 2 we recall the pricing of index and tranche swaps from Errais, Giesecke, and Goldberg (2006). The loss process is specified in Section 3. In Section 4 we discuss the re-scaling algorithm. The exact algorithm is analyzed in Section 5. Implementation details are explained in Section 6. In Section 7 we consider the calibration to market index and tranche spreads. Section 8 concludes.

\section{FAIR INDEX AND TRANCHE SPREADS}

Uncertainty is modeled by a complete probability space $(\Omega, \mathscr{F}, P)$, where $P$ is a pricing measure with respect to a constant risk-free rate of interest $r>0$. Consider a portfolio of $n$ credit sensitive positions. The default process $N$ counts the number of defaults. It has a jump of size 1 at each default time and is otherwise constant. The loss process $L$ records the cumulative financial loss due to default. It jumps by the magnitude of the loss at each default and is otherwise constant. These processes are adapted to a right continuous and complete filtration $\mathbb{F}=\left(\mathscr{F}_{t}\right)_{t \geq 0}$ that models the evolution of investor information.

A portfolio credit derivative is a contingent claim on $N$ or $L$. It allows investors to trade default insurance on the portfolio. An index swap is based on a portfolio whose $n$ constituent single name swaps have common notional 1 , common maturity date $T$ and common premium payment dates $\left(t_{m}\right)$. The default leg of an index swap is a stream of payments that cover portfolio losses as they occur. Its value at time 0 is given by the discounted cumulative losses

$$
D(T)=E\left[\int_{0}^{T} e^{-r t} d L_{t}\right]
$$

The premium leg of an index swap is a stream of payments at dates $\left(t_{m}\right)$. The cash flow at $t_{m}$ is a fraction $S$ of the total notional on the names that have survived until $t_{m}$. Neglecting premium accruals, the value at time 0 of the premium leg is given by

$$
P(T, S)=S E\left[\sum_{m} e^{-r t_{m}} c_{m}\left(n-N_{t_{m}}\right)\right]
$$

where $c_{m}$ is the appropriate day count fraction for the period $m$. The fair index swap spread $S$ is the solution $S=S(T)$ to the equation $D(T)=P(T, S)$.

Investors interested in narrower risk profiles invest in contracts based on a tranche of the index specified by a lower attachment point $\underline{K} \in[0,1]$ and an upper attachment point $\bar{K} \in(\underline{K}, 1]$. The product of the difference $K=\bar{K}-\underline{K}$ and the portfolio notional $n$ is the tranche notional.

The default leg of a tranche swap is a stream of payments that cover portfolio losses as they occur, given that the cumulative losses are larger than $\underline{K} n$ but do not exceed $\bar{K} n$.
The cumulative payments at time $t$, denoted $U_{t}$, are

$$
U_{t}=\left(L_{t}-\underline{K} n\right)^{+}-\left(L_{t}-\bar{K} n\right)^{+} .
$$

The value of these payments at time 0 is

$$
D(\underline{K}, \bar{K}, T)=E\left[\int_{0}^{T} e^{-r t} d U_{t}\right] .
$$

This formula is analogous to formula (1) for the value of an index swap default leg. The latter can be viewed as the default leg of a tranche swap for which $\underline{K}=0$ and $\bar{K}=1$.

The premium leg of a tranche swap has two parts. The first part is an upfront payment, which is expressed as a fraction $F$ of the tranche notional $K n$. The second part is a stream of payments at dates $\left(t_{m}\right)$. For a tranche with $\bar{K}<1$, the cash flow at $t_{m}$ is a fraction $S$ of the difference between the tranche notional and the tranche loss at $t_{m}$. Neglecting accruals, the value of the premium leg is given by

$$
P(\underline{K}, \bar{K}, T, F, S)=F K n+S E\left[\sum_{m} e^{-r t_{m}} c_{m}\left(K n-U_{t_{m}}\right)\right] .
$$

For a fixed upfront payment rate $F$, the fair tranche spread $S$ is the solution $S=S(\underline{K}, \bar{K}, T, F)$ to the equation $D(\underline{K}, \bar{K}, T)=$ $P(\underline{K}, \bar{K}, T, F, S)$. Similarly, for a fixed tranche spread $S$, the fair tranche upfront rate $F$ is the solution $F=F(\underline{K}, \bar{K}, T, S)$ to the equation $D(\underline{K}, \bar{K}, T)=P(\underline{K}, \bar{K}, T, F, S)$. To assess the mark-to-market or fair spread/upfront rate of an index or tranche we model the default and loss point processes.

\section{DEFAULT AND LOSS PROCESSES}

The default process $N$ is a submartingale in the filtration $\mathbb{F}$. The Doob-Meyer theorem guarantees that $N$ can be decomposed into the sum of a non-decreasing predictable process $A$ starting at 0 and an $\mathbb{F}$-martingale. We assume that $A$ is absolutely continuous with respect to Lebesgue measure with non-negative, $\mathbb{F}$-adapted intensity process $\lambda$. Intuitively, $\lambda_{t}$ is the $\mathscr{F}_{t}$-conditional event arrival rate at time $t$. In a portfolio of $n$ names, $N$ is stopped at $n$ so the intensity jumps to 0 at the $n$th default.

We directly specify the intensity. We assume that $\lambda$ is driven by three sources of randomness: the timing of events, the loss at events and a standard Brownian motion $W$ that evolves independently of $L$ and is adapted to $\mathbb{F}$. More precisely, on the event $\left\{N_{t}<n\right\}$ the process $\lambda$ satisfies the stochastic differential equation

$$
d \lambda_{t}=\kappa\left(c-\lambda_{t}\right) d t+\sigma \sqrt{\lambda_{t}} d W_{t}+\delta d L_{t}
$$

with $\lambda_{0}>0$. At an event, the loss process $L$ jumps and so does the intensity. The jump size is the product of the loss and the sensitivity parameter $\delta \geq 0$. Each loss is drawn 


\section{Giesecke and Kim}

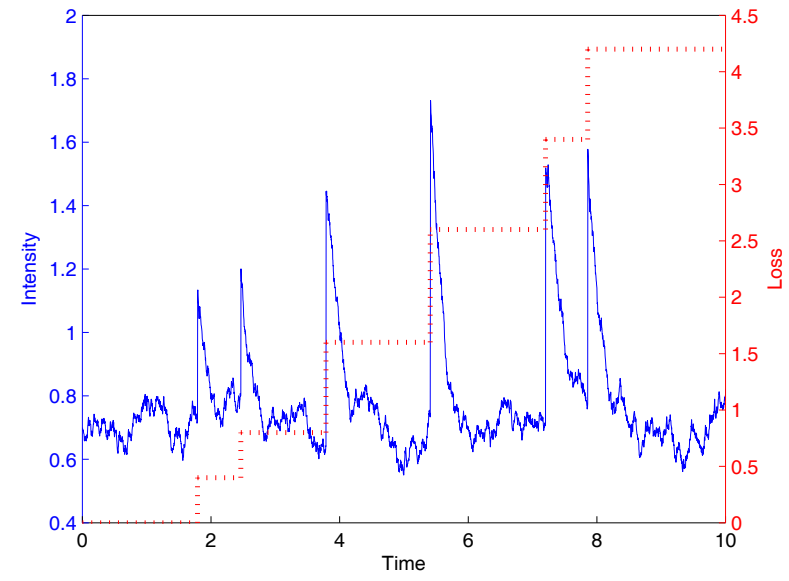

Figure 1: Sample paths of the intensity (5) and the loss process generated with the re-scaling algorithm described in Section 4. A jump in the intensity represents the impact of a default. The jump size is the product of the loss at default and the sensitivity parameter $\delta=1$. The loss at default is drawn from an independent uniform distribution on $\{0.4,0.6,0.8,1\}$. The reversion rate $\kappa=5$ and the reversion level $c=\lambda_{0}=0.7$. The volatility $\sigma=0.2$ controls the diffusive fluctuation of the intensity between events. The portfolio contains $n=100$ names.

independently of previous losses as well as $N$ and $W$ from a distribution $v$ on $(0,1]$. After the event the intensity reverts back to the level $c$ exponentially in mean at a rate $\kappa \geq 0$, with diffusive fluctuations that are driven by the Brownian motion $W$. The parameter $\sigma \geq 0$ describes the diffusive volatility of the intensity. If $2 \kappa c \geq \sigma^{2}$, then $\lambda_{t}>0$ almost surely. Sample paths of the loss process and the intensity are in Figure 1. The product formula shows that equation (5) has solution

$$
\begin{aligned}
\lambda_{t}= & c+\left(\lambda_{0}-c\right) e^{-\kappa t}+\sigma \int_{0}^{t} e^{-\kappa(t-s)} \sqrt{\lambda_{s}} d W_{s} \\
& +\delta \int_{0}^{t} e^{-\kappa(t-s)} d L_{s} .
\end{aligned}
$$

The arrival intensity depends on the loss process $L$ itself. It follows that $L$ is self-affecting. This property captures the feedback effects of a default. Moreover, the intensity and the recovery rate are negatively correlated. Both event feedback (contagion) and negative dependence between default and recovery rates are empirically well documented phenomena, see Jorion and Zhang (2006) and Altman et al. (2005), respectively.

The model (5) nests several classical models. If $\sigma=0$, then $L$ is a compound Hawkes (1971) process stopped at the $n$th arrival, which is a stopped linear birth process if $\kappa=0$. If $\delta=0$, then $L$ is a stopped compound point process that is doubly stochastic. This means that conditional on a path of $W, L$ is a stopped inhomogeneous Poisson process. Up to the $n$th event, the intensity follows a Feller (1951) diffusion, which in finance is also known as a Cox, Ingersoll, and Ross (1985) process.

The intensity model (5) can be generalized to multiple dimensions, to include coefficients that are deterministic functions of time, and to include additional jump terms. The associated point process belongs to the family of affine point processes. Errais, Giesecke, and Goldberg (2006) show that the conditional characteristic function of an affine point process is exponentially affine in the intensity, with coefficient functions satisfying a system of Riccati ordinary differential equations. A call option $E\left[\left(L_{t}-K\right)^{+}\right]$on the loss can then be valued numerically by Fourier inversion. Call values for different strikes $K$ and maturities $t$ determine the fair spread on a tranche.

In this article we estimate index and tranche spreads by evaluating the expectations (1)-(4) using Monte Carlo simulation of the default and loss point processes. A replication consists of the sequence $\left(T^{k}, \ell^{k}\right)$ of ordered default times $0=T^{0}<T^{1}<T^{2}<\ldots$ on $[0, T]$ and loss magnitudes $\ell^{k}$. The associated default and loss process paths are given by the sums $N_{t}=\sum_{k=1}^{n} 1\left(T^{k} \leq t\right)$ and $L_{t}=\sum_{k=1}^{n} \ell^{k} 1\left(T^{k} \leq t\right)$. Below we describe alternative approaches to generate a replication. Each approach exploits the specification of the loss process through the intensity (5).

\section{TIME CHANGE}

Since the default process $N$ has intensity $\lambda$, the compensator $A_{t}=\int_{0}^{t} \lambda_{s} d s$ is continuous. The intensity $\lambda_{t}$ is strictly positive on the event $\left\{N_{t}<n\right\}$ and jumps to 0 at the $n$th default. Therefore, $A$ is strictly increasing on $\left\{N_{t}<n\right\}$ and constant on $\left\{N_{t} \geq n\right\}$. Let $A_{\infty}=\lim _{t \rightarrow \infty} A_{t}$. For $s \in\left[0, A_{\infty}\right)$, define $A_{s}^{-1}=\inf \left\{t: A_{t}>s\right\}$. The process $A^{-1}$ is continuous and strictly increasing. It follows that each $A_{s}^{-1}$ is a finite $\mathbb{F}$-stopping time. The time change theorem of Meyer (1971) implies that the process $N_{A^{-1}}$ is a standard Poisson process on $\left[0, A_{\infty}\right)$ in the right continuous, time-changed filtration $\mathbb{G}$ generated by the stopping time sigma-fields $\mathscr{F}_{A_{s}^{-1}}$ for $s \in\left[0, A_{\infty}\right)$. Therefore, with $T^{k}$ denoting the $k$ th default time, the random variables

$$
S^{k}=A_{T^{k}}=\int_{0}^{T^{k}} \lambda_{s} d s, \quad k=1,2, \ldots, n
$$

are the arrival times of a standard $\mathbb{G}$-Poisson process in $\left[0, A_{\infty}\right)$. The inter-arrival times $S^{k}-S^{k-1}$ are mutually independent, standard exponential random variables. Conversely (see Giesecke and Tomecek 2005), the $k$ th default time is the hitting time of $A$ to the random level $S^{k}$ : almost surely,

$$
T^{k}=A_{S^{k}}^{-1}=\inf \left\{t: \int_{0}^{t} \lambda_{s} d s>S^{k}\right\}
$$




\section{Giesecke and Kim}

To simulate $L$ by re-scaling of Poisson arrivals we need to generate a trajectory of the intensity $\lambda$. If $\delta>0$, the intensity is updated at an event so the path must be generated piece-wise between arrivals. From equation (5), the $k$ th inter-arrival intensity $h$ follows the Feller diffusion

$$
d h_{t}=\kappa\left(c-h_{t}\right) d t+\sigma \sqrt{h_{t}} d W_{t}
$$

started at $h_{0}=\lambda_{T^{k-1}}$. The condition $2 \kappa c \geq \sigma^{2}$ guarantees that $h_{t}>0$ almost surely. In case $\sigma=0, h$ is deterministic. If $\sigma>0$, Euler discretization of the SDE (7) can be used to approximate the path on a discrete-time grid $\left[0=t_{0}<\right.$ $\left.t_{1}<\cdots<t_{M}=t\right]$ with equal spacing $\Delta t$. This scheme is easy to implement, but the probability of getting a negative value for $h_{t}$ is strictly positive. An alternative is to sample directly from the transition law of $h_{t_{i}}$ given $h_{t_{i-1}}$. As shown by Cox, Ingersoll, and Ross (1985), up to a scale factor, the distribution of $h_{s}$ given $h_{t}$ is non-central chi-squared. For $x \geq 0, y, \sigma>0$ and $0 \leq t<s$, the density $g(x ; y, s-t) d x=$ $P\left[h_{s} \in d x \mid h_{t}=y\right]$ is given by

$$
g(x ; y, s)=a e^{-(a x+b y)}\left(\frac{a x}{b y}\right)^{\frac{q}{2}} I_{q}(2 \sqrt{a x b y})
$$

where $I_{q}$ is the modified Bessel function of the first kind of order $q=2 \kappa c / \sigma^{2}-1$ and

$$
\begin{aligned}
& a=2 \kappa /\left(\sigma^{2}(1-\exp (-\kappa s))\right) \\
& b=a \exp (-\kappa s) .
\end{aligned}
$$

The following re-scaling algorithm generates the $k$ th default time and loss and the intensity at the $k$ th default given the previous default time $T^{k-1}(\omega)$ and intensity $\lambda_{T^{k-1}}(\omega)$ :

1. Draw a standard uniform variate $U(\omega)$.

2. Generate a path $h(\omega)$ of the inter-arrival intensity with $h_{0}(\omega)=\lambda_{T^{k-1}}(\omega)$ and set

$$
\begin{aligned}
T^{k}(\omega)= & T^{k-1}(\omega) \\
& +\inf \left\{t: \int_{0}^{t} h_{s}(\omega) d s>-\log U(\omega)\right\} .
\end{aligned}
$$

3. Draw $\ell^{k}(\omega)$ from the distribution $v$ on $(0,1]$.

4. Set $\lambda_{T^{k}}(\omega)=h_{T^{k}-T^{k-1}}(\omega)+\ell^{k}(\omega)$.

Figure 1 shows a sample path of the loss process and the intensity generated with the re-scaling algorithm, and Figure 2 shows the associated path of the compensator, Poisson arrivals and default times. Step (ii) is implemented as follows. The inter-arrival intensity values $h_{t_{i}}(\omega)$ on the discrete-time grid with spacing $\Delta t=1 / 365$ are generated by sampling from the chi-squared density using the method described in Section 3.1 of Broadie and Kaya (2006). The

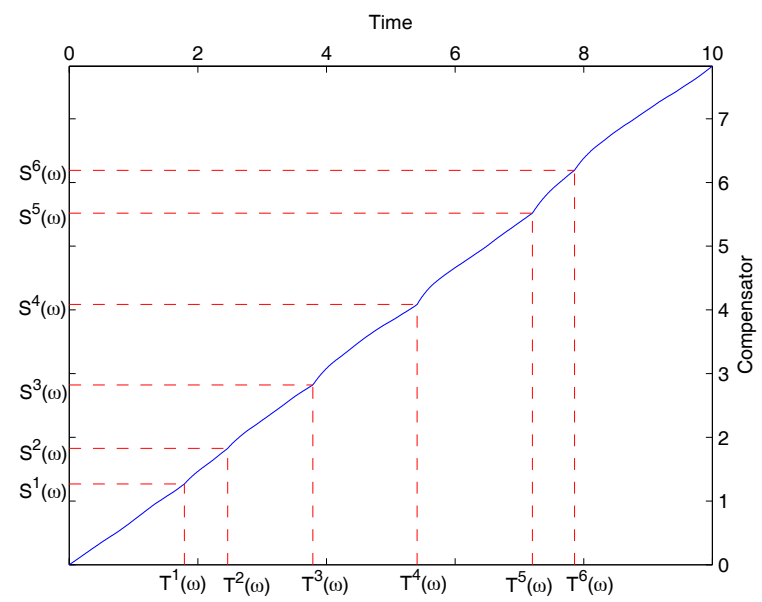

Figure 2: Sample paths of the compensator $A$, standard Poisson arrival times $S^{k}$ and default times $T^{k}$ associated with the intensity and loss process paths shown in Figure 1. The compensator path indicates the response of the intensity to events.

corresponding values of the integrated inter-arrival intensity are given by the running sums of the terms $h_{t_{i}}(\omega)\left(t_{i}-t_{i-1}\right)$. The inter-arrival time $T^{k}(\omega)-T^{k-1}(\omega)$ is the first grid time point $t_{i}$ at which the running sum exceeds $-\log U(\omega)$. If $T$ is the horizon, we stop if $t_{i}>T-T^{k-1}(\omega)$. Then $T^{k-1}(\omega)$ is the last default time in $[0, T]$. The simulation environment is described in Section 6.

\section{EXACT SIMULATION}

Unless the diffusive volatility $\sigma=0$, the simulation of the intensity path is computationally intensive and introduces discretization bias. It can be avoided by observing that given $\mathscr{F}_{T^{k-1}}$, the waiting time $T^{k}-T^{k-1}$ to the next default is the first arrival time of a doubly stochastic counting process with intensity $h$, started at $T^{k-1}$ with $h_{0}=\lambda_{T^{k-1}}$. The doubly stochastic property and the strong Markov property of the inter-arrival intensity $h$ imply that the conditional survival function of the waiting time is given by

$$
P\left[T^{k}-T^{k-1}>t \mid \mathscr{F}_{T^{k-1}}\right]=p\left(t ; \lambda_{T^{k-1}}\right)
$$

where for $t \geq 0$ and $y>0$,

$$
p(t ; y)=E\left[e^{-\int_{0}^{t} h_{s} d s} \mid h_{0}=y\right] .
$$

We observe that $p(t ; y)$ is the price at time 0 of a zero coupon bond maturing at time $t$ if the short term interest rate follows the process $h$ and the initial short rate is $h_{0}=y$. But $h$ is governed by the Feller diffusion (7), so

$$
p(t ; y)=e^{\alpha(t)+\beta(t) y} .
$$




\section{Giesecke and Kim}

For $\sigma=0$, the inter-arrival intensity $h$ is deterministic and the coefficient functions can be obtained by a simple integration. For $\sigma>0$ the coefficient functions are given by

$$
\begin{aligned}
& \alpha(t)=\frac{\kappa c}{\sigma^{2}}\left(\kappa t-2 \log \frac{\cosh \left(-\frac{1}{2} \gamma t+\operatorname{atanh}(-\kappa / \gamma)\right)}{\cosh (\operatorname{atanh}(-\kappa / \gamma))}\right) \\
& \beta(t)=\frac{1}{\sigma^{2}}\left(\kappa+\gamma \tanh \left(-\frac{1}{2} \gamma t+\operatorname{atanh}(-\kappa / \gamma)\right)\right)
\end{aligned}
$$

where $\gamma=\sqrt{\kappa^{2}+2 \sigma^{2}}$; see Cox, Ingersoll, and Ross (1985).

Given the conditional survival function $p(t ; y)$ and an intensity value $\lambda_{T^{k-1}}$ we can simulate the inter-arrival time $T^{k}-T^{k-1}$ by the inverse method. Conditional on $T^{k}$, we must then generate the intensity $\lambda_{T^{k}}$ in order to simulate the next inter-arrival time $T^{k+1}-T^{k}$. This means we need to sample from the conditional distribution of $h_{\tau}$ given $h_{0}$ and $\tau$, where $\tau$ is the first arrival time of a doubly stochastic counting process with intensity $h$. Consider the corresponding conditional density $c(x ; y, t) d x=P\left[h_{\tau} \in d x \mid h_{0}=y, \tau=t\right]$. For $x \geq 0, y>0$ and $t \geq 0$, by Bayes' rule we have

$$
c(x ; y, t)=\frac{q(x, t ; y)}{-\partial_{t} p(t ; y)}
$$

where $q(x, t ; y) d x d t=P\left[h_{\tau} \in d x, \tau \in d t \mid h_{0}=y\right]$ is the joint conditional density of $\left(h_{\tau}, \tau\right)$, and $-\partial_{t} p(t ; y) d t=P[\tau \in$ $\left.d t \mid h_{0}=y\right]$ is the conditional density of $\tau$. By Bayes' rule and the doubly stochastic property of $\tau$, we get

$$
q(x, t ; y)=x g(x ; y, t) E\left[e^{-\int_{0}^{t} h_{s} d s} \mid h_{0}=y, h_{t}=x\right]
$$

where $g(x ; y, t)$ is the non-central chi-squared density (8). The conditional expectation on the right hand side of equation (14) can be calculated explicitly if $h$ follows a Feller diffusion; see Broadie and Kaya (2006) for a formula. We sample from $c(x ; y, t)$ by the inverse method.

The following exact algorithm generates the $k$ th default time and loss and the intensity at the $k$ th default given the previous default time $T^{k-1}(\omega)$ and intensity $\lambda_{T^{k-1}}(\omega)$ :

1. Draw a standard uniform variate $U(\omega)$ and set

$$
\begin{aligned}
T^{k}(\omega)= & T^{k-1}(\omega) \\
& +\inf \left\{t: p\left(t ; \lambda_{T^{k-1}}(\omega)\right) \leq U(\omega)\right\} .
\end{aligned}
$$

2. If $\sigma>0$, draw $h_{T^{k}-T^{k-1}}(\omega)$ from the conditional density $c\left(x ; \lambda_{T^{k-1}}(\omega), T^{k}(\omega)-T^{k-1}(\omega)\right)$ where $c(x ; y, s)$ is given by equation (13).

3. Draw $\ell^{k}(\omega)$ from the distribution $v$ on $(0,1]$.

4. Set $\lambda_{T^{k}}(\omega)=h_{T^{k}-T^{k-1}}(\omega)+\ell^{k}(\omega)$.

Figure 3 shows a path of the loss process generated with the exact algorithm, along with the corresponding

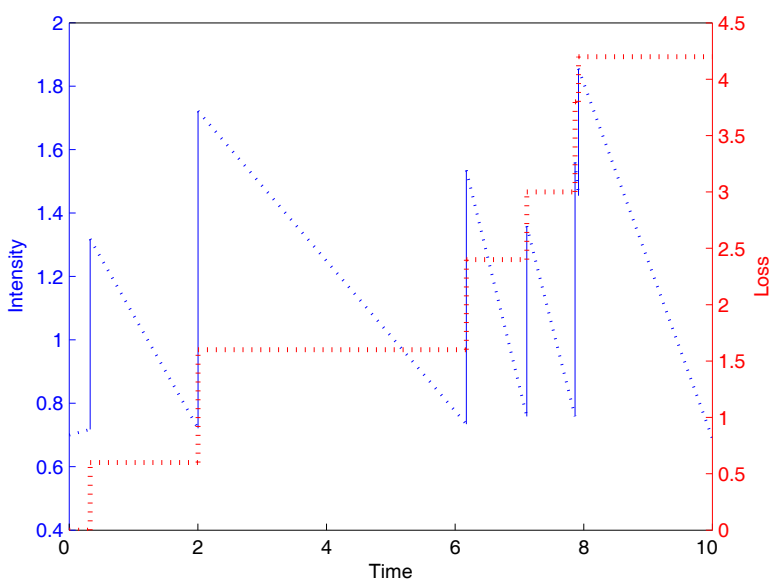

Figure 3: Sample path of the loss process generated with the exact algorithm, along with the corresponding intensity values just before and at the default times. The difference between the intensity values just before and at an event is equal to the financial loss at the event. The parameter values are those used to generate Figures 1 and 2.

intensity values just before and at the default times. Step (i) is implemented as follows. Given the horizon $T$, we first evaluate $p\left(T-T^{k-1}(\omega) ; \lambda_{T^{k-1}}(\omega)\right)$. If this value is larger than $U(\omega)$ we stop. In this case $T^{k-1}(\omega)$ is the last default time in $[0, T]$. Otherwise, we numerically solve $p\left(t ; \lambda_{T^{k-1}}(\omega)\right)-U(\omega)=0$ for $t \in\left[0, T-T^{k-1}(\omega)\right]$ using Brent's method, which combines root bracketing, bisection and inverse quadratic interpolation; see Chapter 4 in Brent (1973). The solution is equal to $T^{k}(\omega)-T^{k-1}(\omega)$.

The exact algorithm extends to the class of self-affecting intensity models for which the conditional inter-arrival time distribution $p(t ; y)$ can be calculated and the conditional distribution of the inter-arrival intensity $h$ is known. For example, the inter-arrival intensity $h$ could follow a general affine jump diffusion, in which case $p(t ; y)$ retains the exponentially affine form (12); see Duffie, Pan, and Singleton (2000). Another example is a quadratic diffusion model, in which $\log p(t ; y)$ is a quadratic function of $y$; see Leippold and $\mathrm{Wu}$ (2002). The price of this generality is that, except for special cases, the ordinary differential equations that govern the coefficients do not admit closed form solutions and the conditional distribution of $h$ is known only through its characteristic function. In this situation Steps (i) and (ii) are computationally intensive.

\section{IMPLEMENTATION}

The simulation experiments in this paper were performed on a desktop PC with an AMD Athlon $1.00 \mathrm{GHz}$ processor and 960 MB of RAM, running Windows XP Professional. The codes were written in $\mathrm{C}++$ and a Windows .NET application was built. The compiler used was Microsoft Visual C++ .NET version 7.1.3088. For random number generation, the 
evaluation of special functions and Brent's method we used the code provided in the GNU Scientific Library. Table 1 compares the performance of the two algorithms in this environment.

Table 1: Comparison of simulation algorithms. The table shows the estimated spread on a 5 year index swap with $n=100$ names (basis points), the $95 \%$ confidence intervals estimated by bootstrapping (basis points) and the calculation time (seconds). The parameter values are $r=0.05, \delta=1, \kappa=5, c=\lambda_{0}=0.7, \sigma=0.2$, and the loss at default is drawn from an independent uniform distribution on $\{0.4,0.6,0.8,1.0\}$. 100,000 replications were generated.

\begin{tabular}{rlll}
\hline Algorithm & Spread & $95 \%$ CI & Time \\
\hline Re-scaling & 58.02 & {$[57.79,58.25]$} & 54.17 \\
Exact & 58.18 & {$[57.94,58.41]$} & 27.15 \\
\hline
\end{tabular}

\section{CALIBRATION}

We calibrate the intensity model (5) and the distribution $v$ of the loss at default to market index and tranche spreads/upfront rates on the CDX North America High Yield index observed on 5/11/2007. The data were obtained from Morgan Stanley. The CDX.NA.HY index has $n=100$ names. The attachment points are $0,10,15,25,35$ and 100 percent. The $[0,10]$ and $[10,15]$ tranches are quoted in terms of a percentage upfront fee. The other tranches are quoted in terms of a running spread. We do not use the $[35,100]$ (super senior) tranche in the calibration.

We assume $v$ is uniform on $\left\{\ell_{1}, \ell_{2}\right\}$ with $0<\ell_{1}<\ell_{2}<1$. Consistent with market practice, we set the expected loss at default $\int z d v(z)=0.6$. With this condition $v$ is specified by one parameter, $\ell_{1}$ say. The intensity $\lambda$ is specified by the parameters $\lambda_{0}, c, \kappa, \sigma$ and $\delta$. The risk-free rate $r=0.05$.

We fit the parameter vector $\theta=\left(\lambda_{0}, c, \kappa, \sigma, \delta, \ell_{1}\right)$ to the CDX.NA.HY market data. Using adaptive simulated annealing, we numerically solve the optimization problem

$$
\min _{\theta \in \Theta} \sum_{i}\left(\frac{\operatorname{MarketMid}(\mathrm{i})-\operatorname{Model}(\mathrm{i}, \boldsymbol{\theta})}{\operatorname{MarketAsk}(\mathrm{i})-\operatorname{MarketBid}(\mathrm{i})}\right)^{2}
$$$$
\text { subject to } 2 \kappa c \geq \sigma^{2}
$$

where $\Theta=[0,5]^{3} \times[0,1] \times[0,5] \times[0.2,0.6]$ and the sum ranges over the data points. The market mid quote MarketMid is the arithmetic average of the observed MarketAsk and MarketBid quotes. The model value Model is a function of $\theta$; see Sections 2 and 3 .

We analyze two model specifications: a model Mod 1 which is unrestricted and a restricted model Mod 2 for which the diffusive volatility $\sigma=0$. In Mod 2, the loss process $L$ is a stopped compound Hawkes process with deterministic inter-arrival intensity.

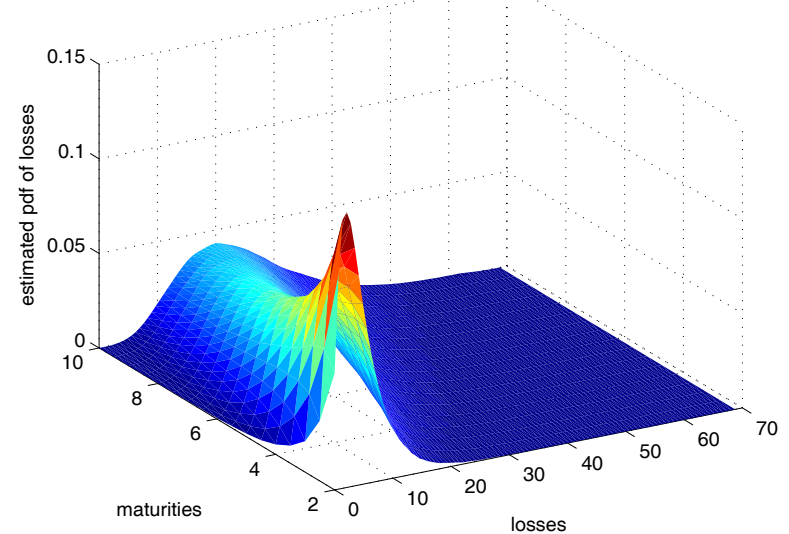

Figure 4: Loss distribution for different horizons implied by the index and tranche spreads on the 5Y CDX.NA.HY index observed on 5/11/2007. We simulate the loss process from the model Mod 1 with parameters set to the calibrated values given in Table 3 and then use kernel smoothing to non-parametrically estimate the loss distribution. The mass in the tail of the distribution increases with the horizon.

We begin by calibrating to quotes for a single maturity. The data and results are summarized in Table 2 . Both specifications fit the data well; the unrestricted model (average absolute percentage error AAPE 1.47\%) does better than the restricted model (AAPE 2.24\%). The calibrated parameter values for the two models are very similar, see Table 3, and so are the implied loss distributions. Figure 4 shows the loss distribution for different horizons implied by the unrestricted model with parameters set to the calibrated values in Table 3.

Table 2: Calibration data and results. The CDX.NA.HY $5 Y$ market quotes are observed on 5/11/2007. They are in basis points except for the $[0,10]$ and $[10,15]$ tranches, which are quoted in terms of a percentage upfront fee. 100,000 replications were generated. The values in column Mod 1 refer to spreads generated by the unrestricted model while the values in column Mod 2 refer to model spreads when $\sigma=0$. We report the minimum value of the objective function MinObj and the average absolute percentage error AAPE.

\begin{tabular}{rllll}
\hline & MarketBid & MarketAsk & Mod 1 & Mod 2 \\
\hline $0-10$ & $70.50 \%$ & $70.75 \%$ & $71.11 \%$ & $71.48 \%$ \\
$10-15$ & $34.25 \%$ & $34.50 \%$ & $32.85 \%$ & $32.74 \%$ \\
$15-25$ & 316.00 & 319.00 & 316.80 & 311.43 \\
$25-35$ & 79.00 & 81.00 & 81.47 & 77.34 \\
Index & 262.85 & 263.10 & 263.46 & 262.97 \\
\hline MinObj & & & 41.63 & 60.41 \\
AAPE & & & $1.47 \%$ & $2.24 \%$ \\
\hline
\end{tabular}

To assess the stability of the calibrations over time we re-calibrate both specifications at different dates starting at 


\section{Giesecke and Kim}

Table 3: Initial and calibrated parameter values for the unrestricted model Mod 1 and the restricted model Mod 2. We ran several calibrations with different initial values. The setting we report here had the lowest objective function value for model Mod 1.

\begin{tabular}{rllllll}
\hline & $\lambda_{0}$ & $c$ & $\kappa$ & $\sigma$ & $\delta$ & $\ell_{1}$ \\
\hline Initial & 2.50 & 2.50 & 2.50 & 0.50 & 2.50 & 0.40 \\
Mod 1 & 0.70 & 1.61 & 2.62 & 0.62 & 2.99 & 0.24 \\
Mod 2 & 0.75 & 1.60 & 2.58 & 0.00 & 2.94 & 0.24 \\
\hline
\end{tabular}

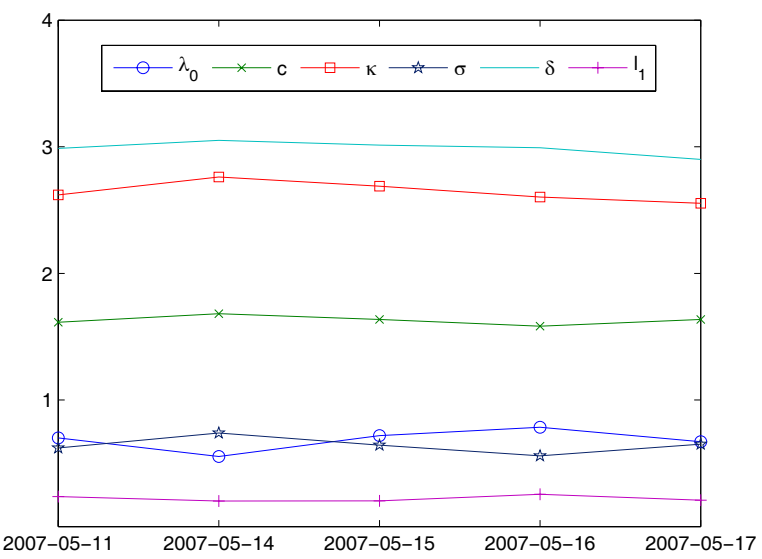

Figure 5: Parameter stability: calibrated parameter values for the unrestricted model Mod 1 at different calibration dates.

$5 / 11 / 2007$. The initial values at a given date after 5/11 are set to the optimal values obtained at the previous calibration date. Figure 5 (Figure 6) shows the calibrated parameter values for the unrestricted model Mod 1 (the restricted model Mod 2) for the different dates. The calibrated parameter values for the unrestricted model are more stable than the values for the restricted model. Table 4 (Table 5) gives the calibration errors. In each calibration, the unrestricted model leads to a lower average absolute percentage error than the restricted model.

Table 4: Comparison of the calibrations of the unrestricted model Mod 1 at the different calibration dates. The calibrated parameter values are shown in Figure 5.

\begin{tabular}{rlllll}
\hline Date & $05 / 11$ & $05 / 14$ & $05 / 15$ & $05 / 16$ & $05 / 17$ \\
\hline MinObj & 41.63 & 58.66 & 46.78 & 46.24 & 58.24 \\
AAPE & $1.47 \%$ & $1.70 \%$ & $1.26 \%$ & $1.32 \%$ & $1.35 \%$ \\
\hline
\end{tabular}

Finally we examine the fit to quotes for several maturities, all observed on 5/11/2007. We get 5 additional data points per additional maturity. We calibrate simultaneously to 5 and 7 year CDX.NA.HY index and tranche spreads. The data and results are shown in Table 6. The calibrated parameter values are given in Table 7 . For both model specifications the average absolute percentage error

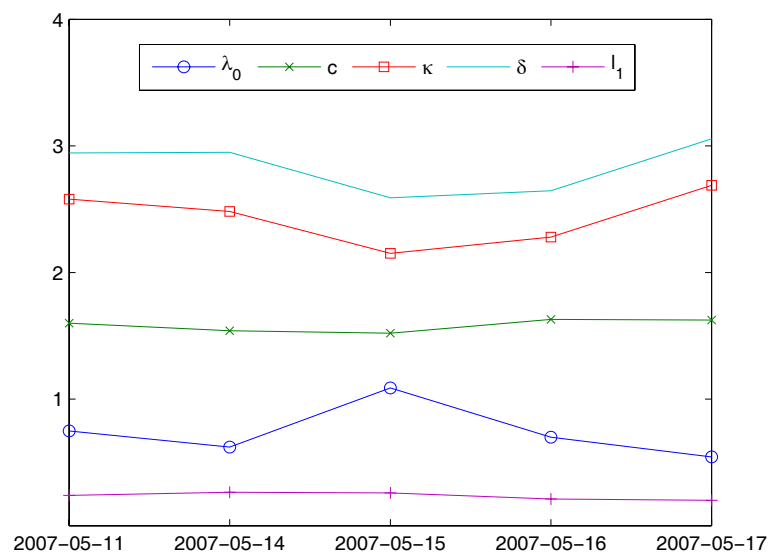

Figure 6: Parameter stability: calibrated parameter values for the restricted model Mod 2 at different calibration dates.

Table 5: Comparison of the calibrations of the restricted model Mod 2 at the different calibration dates. The calibrated parameter values are shown in Figure 6.

\begin{tabular}{rlllll}
\hline Date & $05 / 11$ & $05 / 14$ & $05 / 15$ & $05 / 16$ & $05 / 17$ \\
\hline MinObj & 60.41 & 73.43 & 54.29 & 43.88 & 65.23 \\
AAPE & $2.24 \%$ & $2.04 \%$ & $1.57 \%$ & $1.45 \%$ & $1.92 \%$ \\
\hline
\end{tabular}

increases somewhat, but the fits are still very reasonable. The unrestricted model (AAPE 2.35\%) outperforms the restricted model (AAPE 3.30\%).

\section{CONCLUSION}

We describe two algorithms to simulate a loss point process that is specified in terms of an intensity. The re-scaling algorithm requires the simulation of intensity paths. Path simulation is time-consuming and introduces bias into the results. The exact algorithm avoids path simulation and generates an unbiased estimate of the derivative price. While the exact algorithm requires much less time per replication than the re-scaling algorithm, speed may still be an issue for some applications. An example is model calibration, which requires a large number of price evaluations at different parameters values. The bottleneck in the exact algorithm is the numerical evaluation of the inverse of the conditional distribution of the inter-arrival time. At present we are investigating a thinning algorithm that avoids this step.

\section{ACKNOWLEDGMENTS}

We would like to thank Lisa Goldberg and Jeremy Staum for useful comments and suggestions. We are grateful to Morgan Stanley for providing the index and tranche market data. 
Table 6: Extended calibration: data and results. The CDX.NA.HY 5Y and 7Y market quotes are observed on $5 / 11 / 2007$. They are in basis points except for the $0-10$ and 10-15 percent tranches, which are quoted in terms of a percentage upfront fee. The first 5 rows are for the $5 \mathrm{Y}$ index while rows 5-10 are for the $7 \mathrm{Y}$ index. 100,000 replications were generated. The values in column Mod 1 refer to spreads generated by the unrestricted model while the values in column Mod 2 refer to model spreads when $\sigma=0$. We report the minimum value of the objective function MinObj and the average absolute percentage error AAPE.

\begin{tabular}{rllll}
\hline & MarketBid & MarketAsk & Mod 1 & Mod 2 \\
\hline $0-10$ & $70.50 \%$ & $70.75 \%$ & $71.69 \%$ & $72.00 \%$ \\
$10-15$ & $34.25 \%$ & $34.50 \%$ & $33.44 \%$ & $33.47 \%$ \\
$15-25$ & 316.00 & 319.00 & 316.13 & 309.93 \\
$25-35$ & 79.00 & 81.00 & 78.45 & 72.79 \\
Index & 262.85 & 263.10 & 263.94 & 262.75 \\
\hline $0-10$ & $80.13 \%$ & $80.38 \%$ & $81.23 \%$ & $81.49 \%$ \\
$10-15$ & $55.50 \%$ & $55.75 \%$ & $55.17 \%$ & $55.40 \%$ \\
$15-25$ & 582.00 & 587.00 & 580.25 & 584.63 \\
$25-35$ & 180.00 & 183.00 & 206.41 & 207.98 \\
Index & 307.50 & 307.75 & 307.53 & 308.91 \\
\hline MinObj & & & 136.12 & 192.82 \\
AAPE & & & $2.35 \%$ & $3.30 \%$ \\
\hline
\end{tabular}

Table 7: Extended calibration: initial and calibrated parameter values for the unrestricted model Mod 1 and the restricted model Mod 2. We used the same initial values as in the basic calibration.

\begin{tabular}{rllllll}
\hline & $\lambda_{0}$ & $c$ & $\kappa$ & $\sigma$ & $\delta$ & $\ell_{1}$ \\
\hline Initial & 2.50 & 2.50 & 2.50 & 0.50 & 2.50 & 0.40 \\
Mod 1 & 0.13 & 1.77 & 2.10 & 0.69 & 2.44 & 0.25 \\
Mod 2 & 0.12 & 1.76 & 1.96 & 0.00 & 2.32 & 0.24 \\
\hline
\end{tabular}

\section{REFERENCES}

Altman, E., B. Brady, A. Resti, and A. Sironi. 2005. The link between default and recovery rates: Theory, empirical evidence and implications. Journal of Business 78 (6): 2203-2227.

Arnsdorf, M., and I. Halperin. 2007. BSLP: Markovian bivariate spread-loss model for portfolio credit derivatives. Working Paper, Quantitative Research J.P. Morgan.

Brent, R. 1973. Algorithms for minimization without derivatives. Englewood Cliffs: Prentice Hall.

Brigo, D., A. Pallavicini, and R. Torresetti. 2006. Calibration of CDO tranches with the dynamical generalizedPoisson loss model. Working Paper, Banca IMI.

Broadie, M., and O. Kaya. 2006. Exact simulation of stochastic volatility and other affine jump diffusion processes. Operations Research 54 (2): 217-231.
Cox, J., J. Ingersoll, and S. Ross. 1985. A theory of the term structure of interest rates. Econometrica 53:385-408.

Davis, M., and V. Lo. 2001. Modeling default correlation in bond portfolios. In Mastering Risk Volume 2: Applications, ed. C. Alexander, 141-151. Prentice Hall.

Ding, X., K. Giesecke, and P. Tomecek. 2006. Time-changed birth processes and multi-name credit. Working Paper, Stanford University.

Duffie, D., and N. Garleanu. 2001. Risk and valuation of collateralized debt obligations. Financial Analysts Journal 57 (1): 41-59.

Duffie, D., J. Pan, and K. Singleton. 2000. Transform analysis and asset pricing for affine jump-diffusions. Econometrica 68:1343-1376.

Duffie, D., and K. J. Singleton. 1998. Simulating correlated defaults. Working Paper, GSB, Stanford University.

Errais, E., K. Giesecke, and L. Goldberg. 2006. Pricing credit from the top down with affine point processes. Working Paper, Stanford University.

Feller, W. 1951. Two singular diffusion problems. Annals of Mathematics 54:173-182.

Giesecke, K., and L. Goldberg. 2005. A top down approach to multi-name credit. Working Paper, Stanford University.

Giesecke, K., and P. Tomecek. 2005. Dependent events and changes of time. Working Paper, Stanford University.

Hawkes, A. G. 1971. Spectra of some self-exciting and mutually exciting point processes. Biometrika 58 (1): 83-90.

Jorion, P., and G. Zhang. 2006. Good and bad credit contagion: Evidence from credit default swaps. Journal of Financial Economics, Forthcoming.

Leippold, M., and L. Wu. 2002. Asset pricing under the quadratic class. Journal of Financial and Quantitative Analysis 37:271-295.

Longstaff, F., and A. Rajan. 2006. An empirical analysis of collateralized debt obligations. Forthcoming, Journal of Finance.

Lopatin, A., and T. Misirpashaev. 2007. Two-dimensional Markovian model for dynamics of aggregate credit loss. Working Paper, Numerix.

Meyer, P.-A. 1971. Démonstration simplifée d'un théorème de Knight. In Séminaire de Probabilités V, Lecture Note in Mathematics 191, 191-195. Springer-Verlag Berlin.

Tavella, D., and M. Krekel. 2006. Pricing nth-to-default credit derivatives in the PDE framework. Working Paper, Octanti Associates.

\section{AUTHOR BIOGRAPHIES}

KAY GIESECKE is an assistant professor in the Department of Management Science and Engineering at Stanford University. He has previously held a position in the School of Operations Research and Information Engineering at Cornell University. His research is in 


\section{Giesecke and Kim}

financial engineering, particularly credit risk modeling. His email is <gieseckelstanford.edu> and his web page is <www.stanford.edu/ giesecke>.

BAEHO KIM is a Ph.D. candidate in the Department of Management Science and Engineering at Stanford University. His research interests include stochastic modeling and computational techniques in financial engineering. His email is <baehokimestanford.edu> and web page is <www.stanford.edu/ baehokim>. 